\title{
Estimating Rear-End Accident Probabilities at Signalized Intersections: An Occurrence-Mechanism Approach
}

by

Yinhai Wang ${ }^{1}$, Hitoshi Ieda ${ }^{2}$, and Fred Mannering ${ }^{3}$

June 24, 2002

${ }^{1}$ Research Associate, Department of Civil and Environmental Engineering, Box 352700, University of

Washington, Seattle, WA 98195-2700; yinhai@u.washington.edu; telephone 206-616-2696; fax

206-543-1543

${ }^{2}$ Professor, Department of Civil Engineering, University of Tokyo, Tokyo, Japan 113-8656;

ieda@trip.t.u-tokyo.ac.jp; telephone +81-3-5841-6119; fax +81-3-5841-8506

${ }^{3}$ Professor and Head, School of Civil Engineering, Purdue University, West Lafayette, IN 47907;

flm@ecn.purdue.edu; telephone 765-494-2158; fax 765-494-0395 


\begin{abstract}
At signalized intersections, rear-end accidents are frequently the predominant accident type. These accidents result from the combination lead-vehicle deceleration and the ineffective response of the following vehicle's driver to this deceleration. This paper mathematically represents this process, by expressing accident probability as the product of the probability of the lead vehicle decelerating and the probability of the driver in the following failing to respond in time to avoid a collision. Using this premise, a model of rear-end accident probabilities is estimated using information on traffic flow, traffic regulations, roadway geometrics and human factors from four-legged signalized intersections in Tokyo, Japan. Estimation findings provide some important preliminary evidence for the development of countermeasures to reduce the frequency of rear-end accidents at signalized intersections.
\end{abstract}

Key words: accident probabilities, rear-end accidents, negative binomial regression, perception/reaction time, signalized intersections, driver behavior 


\section{INTRODUCTION}

Of all accidents in Japan, about $60 \%$ of the total, and $45 \%$ of the fatal accidents occur at intersections. Rear-end accidents are the most common type at signalized intersections in Japan, accounting for $35.4 \%$ of intersection accidents and $21.3 \%$ of all vehicular accidents (Institute for Traffic Accident Research and Data Analysis, 1997). Given these high percentages, the study of intersection accidents is clearly a priority in Japan. As evidence of this, many researchers have studied intersection accidents in recent years, although few have studied rear-end accidents as a specific subset of all intersection accidents. The emphasis of most previous work has focused specifically on modeling relationships between accident frequency (number of accidents over some time period) at intersections and geometric/road-environment elements. Popular modeling methods have included simple linear regression, Poisson regression and negative binomial regression.

From a methodological perspective, as the field of accident frequency modeling has matured, Poisson and negative binomial models have emerged as the preferred methodological alternatives (see Jovanis and Chang, 1986, Miaou et al, 1992, and Poch and Mannering, 1996). A primary problem with the Poisson model is that it restricts the mean and variance of the accident frequency data to be equal. This is often not the case with accident frequency data. Such data are often characterized by over dispersion (the variance of the frequency exceeds its mean). The 
negative binomial model relaxes the mean/variance equality restriction and thus accounts for over dispersed data (Lawless, 1987). It has been successfully applied to predict the frequency of traffic accidents on roadway segments and intersections (Shankar et al, 1995 and Poch and Mannering, 1996).

Another characteristic of accident frequency data is a large number of zeros resulting from not having an accident occur at a specific location in a given time period. Recently, researchers have applied two-state models — one state has near zero accident probability and the other state follows a Poisson or negative binomial counting distribution (see Shankar et al, 1997 and Carson and Mannering, 2001).

While past research has provided valuable methodological insight into accident frequency modeling, few studies have explicitly considered rear-end accident probabilities at intersections. Two exceptions are Hauer et al (1988) and Poch and Mannering (1996). Hauer and his colleagues classified intersection vehicle-to-vehicle accidents into 15 types according to vehicle movements before the collision and analyzed the frequencies of accident types (rear-end, sideswipe, etc.). Their classification approach provided a microscopic perspective to analyzing intersection vehicle-to-vehicle accident frequencies. Poch and Mannering (1996) studied the effects of intersection approach conditions on the accident frequencies using negative binomial regression to study the frequencies of various types of accidents. 
However, a common criticism of most previous accident-frequency studies is that they rarely include considerations of human factors. Because human factors play a very important role in accident occurrence, the absence of human factors in accident modeling is a serious specification error. The intent of this paper is to derive and apply a new model of intersection rear-end accident probabilities.

\section{METHODOLOGY}

As shown in the conceptual flow chart of rear-end accident occurrence provided in Figure 1, rear-end accidents are the result of a lead vehicle's deceleration and the ineffective response of the following vehicle's driver. When a leading vehicle decelerates, it becomes an obstacle vehicle for drivers of following vehicles. The frequency of encountering obstacle vehicles is determined by the occurrence of "disturbances" which are defined as anything that interrupts the smooth flow of traffic, such as signal-disregarding pedestrians, right-turning vehicles (vehicles in Japan, where our data originate, drive on the left side), red lights and so on.

The performance of drivers in following vehicles can be viewed as consisting of three successive components: the perception of changes in the traffic environment; the decision to respond to these changes; and an action. Factors affecting drivers' ability to perceive, decide and 
act determine the effectiveness of drivers' reaction to obstacle vehicles, and thus rear-end accident

probability.

Drivers of following vehicles typically gather the majority of their deceleration information from the brake lights of leading vehicles implying independence between the probability of an encountering an obstacle vehicle and the driver failing to avoid the collision. However, in some instances, the driver of the following vehicle may see the disturbance (by seeing in front of the lead vehicle) before deceleration of the lead vehicle occurs, implying some correlation between the probability of encountering an obstacle vehicle and the driver failing to avoid a collision. To simplify matters, we assume herein that the probability of an encountering an obstacle vehicle $\left(P_{o}\right)$ is independent from the probability of its driver failing to avoid the collision $\left(P_{f}\right)$, giving the rear-end accident probability $(P)$ as,

$$
P=P_{o} \cdot P_{f}
$$

From this expression an estimable model can be derived.

\section{Formulating the Probability of Encountering an Obstacle Vehicle, $\boldsymbol{P}_{o}$}

A vehicle becomes an obstacle for the driver of a following vehicle when it decelerates.

This deceleration can be caused by a variety of emerging disturbances. For modeling purposes, it 
is assumed that occurrence of such disturbances follow a Poisson process. This results in time intervals between deceleration-inducing disturbances that are exponentially distributed (see Mannering and Kilareski, 1998). If there is a disturbance $j$ with occurrence rate $\eta_{j}$ in time $t$, then the density function is,

$$
f(t)=\eta_{j} \mathrm{e}^{-\eta_{j} t} \text { for } t>0
$$

For the exponential distribution, the probability of a disturbance occurring is independent of the time that has transpired since the last disturbance occurred. Because accidents are rare and isolated events, this is a reasonable assumption. However in instances of chain-reaction collisions, this assumption may be questioned because the occurrence of a disturbance will be tied to the occurrence of the preceding disturbance. To avoid this problem, in this study we include only two-vehicle accidents, or the collision of the first two vehicles in an vehicle accident involving more than two vehicles, to avoid this problem. This gives the probability of the driver of a leading vehicle encountering a disturbance $j$ in time $t$ as,

$$
P_{j}=\int_{0}^{t} \eta_{j} \mathrm{e}^{-\eta_{j} t} d t=1-\mathrm{e}^{-\eta_{j} t}
$$

Because any disturbance can cause the driver of a leading vehicle to decelerate, the probability of the driver of a following vehicle encountering an obstacle vehicle is equal to the probability that at least one disturbance occurs within some specified time period. Therefore the formulation for $P_{o}$ can be derived as follows, 


$$
P_{o}=1-\prod_{j}\left(1-P_{j}\right)
$$

Combining equations 3 and 4 , a simpler form for $P_{o}$ can be obtained,

$$
P_{o}=1-\mathrm{e}^{-\sum_{j} \eta_{j} t}
$$

In equation $5, \sum_{j} \eta_{j} t$ should always be positive and influenced by external factors (intersection characteristics, traffic flow, and other factors that may influence disturbance occurrence). The problem then becomes one of selecting a function to account for these external factors. For the purposes of model derivation, and integrating this model with driver failure probabilities, we assume that,

$$
\sum_{j} \eta_{j} t=\mathrm{e}^{\boldsymbol{\beta} \mathbf{X}}
$$

which, from equation 5 gives,

$$
P_{\mathrm{o}}=1-\mathrm{e}^{-\mathrm{e}^{\mathrm{BX}}}
$$

where $\beta$ and $X$ are vectors of estimable coefficients and explanatory variables of disturbance frequency respectively.

\section{Formulating drivers' failure probability $\left(\boldsymbol{P}_{f}\right)$}

Driving is a process of perceiving changes in traffic situations and adjusting vehicle operations to adapt these changing conditions. The time needed for drivers to detect changes and 
the quality of their response determines the probability of their avoiding potential collisions. As noted by Johansson and Rumar (1971), one of the main factors in determining if an accident can be avoided is a driver's perception and reaction time (PRT), which can be regarded as a comprehensive reflection of human-related factors. As is well known, the PRT has a wide range of values depending on the complexity of the problem, the complexity of the solution, and drivers' expectancy of hazards (Bates, 1995).

To incorporate perception/reaction time into a model of drivers' failure probability $\left(P_{f}\right)$, we consider available perception/reaction time (APRT) and needed perception/reaction time (NPRT). APRT refers to the time drivers have to complete their perception/reaction under specific traffic situations and NPRT is the needed perception/reaction time, which is ability-oriented and varies from driver to driver. Drivers cannot avoid collisions if their NPRT is greater than APRT. Therefore, the probability of a driver being involved in a rear-end accident is the probability that NPRT is larger than APRT. If APRT and NPRT are assumed to be random variables with some assumed distribution, drivers' failure probability can be determined.

Before selecting appropriate distributions, the effect of age on NPRT must be considered. While physiological evidence suggests that pure reaction time increases with age, perception times may decrease as a result of driver experience and expectations. For example, while Welford (1977) provided empirical evidence that reaction time increased with age, Olson and Sivak (1986) 
found that old drivers and young drivers had almost the same perception/reaction time under surprise and alerted situations. Thus the net effect of age on NPRT is ambiguous. Given this, we will ignore the effect of driver age on NPRT, although our model could be generalized to take age effects into account if such effects were deemed significant.

In terms of an appropriate probability distribution for NPRT, a normal distribution is an obvious choice. Unfortunately, the normal distribution is computationally cumbersome. Thus, we assume a Weibull distribution because of its empirical flexibility and close approximation to a normal distribution. The Weibull density function is,

$$
f(\alpha, \lambda, t)=\alpha \lambda t^{\alpha-1} \mathrm{e}^{-\lambda t^{\alpha}} \quad \text { for } t>0
$$

where $\alpha$ and $\lambda$ are the shape and scale parameters, respectively. A value of 3.25 for $\alpha$ has been empirically shown to be a value that gives the Weibull distribution a good approximation to the normal distribution (Kao, 1960 and Plait, 1962). To visualize the behavior of the Weibull distribution, Figure 2 illustrates density function curves for various $\lambda$ values with $\alpha$ fixed to be 3.25 .

One of the important tasks for traffic engineers is to maintain an acceptable value of available perception/reaction time (APRT) through geometric design and traffic regulations. Unfortunately, variations in driver skills and changeable traffic/environmental conditions make it difficult to estimate the APRT exactly under varying traffic situations. This has led highway 
engineers to design highways to provide a conservative, constant value of APRT ( 2.5 seconds) for highway design (Mannering and Kilareski, 1998). However, the variation in APRT is potentially a critical element in the occurrence of traffic accidents. To account for this variation, APRT is assumed to be a random variable from a known distribution. Again assuming a Weibull distribution and letting $t_{a}$ represent available perception/reaction time, the density function is,

$$
f\left(\alpha, \gamma, t_{a}\right)=\alpha \gamma t_{a}^{\alpha-1} \mathrm{e}^{-\gamma t_{a}^{\alpha}} \quad \text { for } t_{v}>0
$$

where $\alpha$ and $\gamma$ are the Weibull distribution's shape and scale parameters respectively. Based on these assumptions, drivers' failure probability can be expressed as,

$$
P_{f}=\int_{0}^{\infty} \int_{\mathrm{t}_{a}}^{\infty} f(\alpha, \lambda, t) f\left(\alpha, \gamma, t_{a}\right) d t d t_{a}=\int_{0}^{\infty} \mathrm{e}^{-\lambda t_{a}^{\alpha}} \alpha \gamma t_{a}^{\alpha-1} \mathrm{e}^{-\gamma t_{a}^{\alpha}} d t_{a}=\frac{1}{1+\lambda / \gamma}
$$

Note that as long as $\alpha$ is assumed to be the same for NPRT and APRT, $P_{f}$ is determined only by the ratio of $\lambda$ and $\gamma$. Because parameters $\lambda$ and $\gamma$ are positive, $\lambda / \gamma$ can be related to explanatory variables by using an exponential function,

$$
\lambda / \gamma=e^{-\varphi \mathbf{Z}}
$$

Correspondingly, $P_{f}$ can be rewritten as,

$$
\mathrm{P}_{\mathrm{f}}=\frac{1}{1+e^{-\varphi \mathbf{Z}}}
$$

where $\phi$ and $\mathbf{Z}$ are vectors of estimable coefficients and explanatory variables affecting $P_{f}$, respectively. 


\section{Overall model and model estimation approach}

We wish to develop an expression for a randomly chosen vehicle pair, from through traffic

flow, on leg $k$ of intersection $i$. Referring to the work above, the vehicle-pair rear-end accident probability, $P_{i k}$, can be specified by replacing $P_{o}$ and $P_{f}$ in equation 1 by equations 7 and 12 respectively (and adding subscripts denoting intersection, $i$, and leg, $k$ ) such that,

$$
\mathrm{P}_{\mathrm{ik}}=\mathrm{P}_{\mathrm{oik}} \cdot \mathrm{P}_{\mathrm{fik}}=\frac{1-e^{-e^{\beta \mathbf{X}_{\mathrm{ik}}}}}{1+e^{-\varphi \mathbf{Z}_{\mathrm{ik}}}}
$$

Given this, the number of accidents for some vehicle flow, $v_{i k}$, can be viewed as following a binomial distribution with the probability of having $n_{i k}$ accidents,

$$
P\left(n_{i k}\right)=\left(\begin{array}{c}
v_{i k} \\
n_{i k}
\end{array}\right) P_{i k}^{n_{i k}}\left(1-P_{i k}\right)^{v_{i k}-n_{i k}}
$$

Because accidents are rare events, $P_{i k}$ will be very small and the corresponding traffic volume $v_{i k}$ will be very large for time periods of reasonable length (in the order of months or years). Under such conditions, the Poisson distribution is a good approximation to the binomial distribution (Pitman, 1993) with,

$$
P\left(n_{i k}\right)=\frac{m_{i k}^{n_{i k}} \mathrm{e}^{-m_{i k}}}{n_{i k} !}
$$

where the Poisson distribution parameter is, 


$$
m_{i k}=\mathrm{E}\left(n_{i k}\right)=v_{i k} P_{i k}
$$

As discussed earlier, Poisson models have been extensively used in accident frequency modeling. However, recent studies (see Miaou and Lum, 1993; Shankar et. al., 1995, 1997; Poch and Mannering, 1996; Milton and Mannering, 1998) have demonstrated that the Poisson model is limited in that the requirement that mean and variance of the frequency data be approximately equal is often not met in accident data which tend to be over dispersed (having a variance significantly greater than the mean). The most common approach to overcome this limitation is to add an independently distributed error term, $\varepsilon_{i k}$, to the $\log$ transformation of equation 16 such that,

$$
\ln m_{i k}=\ln \left(v_{i k} P_{i k}\right)+\varepsilon_{\mathrm{ik}}
$$

Assuming that $\exp \left(\varepsilon_{i k}\right)$ is a Gamma distributed variable with mean 1 and variance $\delta$, and substituting equation 17 into equation 15 gives

$$
P\left(n_{i k} \mid \varepsilon_{\mathrm{ik}}\right)=\frac{\mathrm{e}^{\left(-v_{i k} P_{i k} \mathrm{e}^{\varepsilon_{i k}}\right)}\left(v_{i k} P_{i k} \mathrm{e}^{\varepsilon_{i k}}\right)^{n_{i k}}}{n_{i k} !}
$$

Integrating $\varepsilon_{i k}$ out of equation 18 results in a negative binomial distribution,

$$
P\left(n_{i k}\right)=\frac{\Gamma\left(n_{i k}+\theta\right)}{\Gamma\left(n_{i k}+1\right) \Gamma(\theta)}\left(\frac{\theta}{v_{i k} P_{i k}+\theta}\right)^{\theta}\left(\frac{\lambda_{i k} P_{i k}}{v_{i k} P_{i k}+\theta}\right)^{n_{i k}}
$$

where $\theta=1 / \delta$. The mean and variance relationship is now,

$$
\operatorname{Var}\left(n_{i k}\right)=\mathrm{E}\left(n_{i k}\right)\left[1+\delta \mathrm{E}\left(n_{i k}\right)\right]
$$

where $\delta$ is an estimable parameter. The estimation of $\delta$ in equation 20 relaxes the constraint of the 
mean equaling the variance in the Poisson model.

Using equation 13 and equation 19, the overall model can be estimated by standard maximum likelihood methods with the log-likelihood function being,

$$
\mathrm{L}(\boldsymbol{\beta}, \boldsymbol{\varphi}, \theta)=\sum_{i} \sum_{k} \ln \left[\frac{\Gamma\left(\mathrm{n}_{\mathrm{ik}}+\theta\right)}{\Gamma\left(\mathrm{n}_{\mathrm{ik}}+1\right) \Gamma(\theta)}\left(\frac{\theta}{\mathrm{v}_{\mathrm{ik}} \cdot \frac{1-e^{-e^{\beta \boldsymbol{X}_{\mathrm{ik}}}}}{1+e^{-\boldsymbol{\varphi} \mathbf{Z}_{\mathrm{ik}}}}+\theta}\right)^{\theta}\left(\frac{\mathrm{v}_{\mathrm{ik}} \cdot \frac{1-e^{-e^{\beta \boldsymbol{X}_{\mathrm{ik}}}}}{1+e^{-\varphi \mathbf{Z}_{\mathrm{ik}}}}}{1-e^{-e^{\beta \mathrm{X}_{\mathrm{ik}}}}}+\theta\right)^{\mathrm{v}_{\mathrm{ik}} \cdot \frac{\mathrm{n}_{\mathrm{ik}}}{1+\varphi \mathbf{Z}_{\mathrm{ik}}}}+\theta\right.
$$

\section{DATA}

Data were collected by selecting 150 four-legged signalized intersections in the Tokyo Metropolitan area. This selection was based on the considerations of intersection size, surrounding land use patterns, and crossing angles of the approaches (being perpendicular or skewed). The purpose of such a selection process was to arrive at a sample of intersections that typified four-legged intersections in Tokyo.

The data collected for the intersections included the number of accidents on each approach over the four-year time period from 1992 to 1995, daily traffic volume by direction, traffic signal control pattern and other relevant factors. Additional intersection-related data were available from original accident records, which included documents with site figures and specific descriptions relating to observed accidents. Unfortunately, several records were not available or flawed and the 
number of intersections in the sample was reduced to 115.

For our analysis, intersection approaches are used as the unit of observation and the number of accidents occurring on these approaches over the four-year time period from 1992 to 1995 are used. All the applicable rear-end accidents were cataloged according to their movements before the collision, and assigned to the corresponding approach. Unfortunately, a few accident records were missing or incomplete for some rear-end accidents. In cases where police records indicated the presence of rear-end accidents on an approach, and all these accidents could not be confirmed with detailed accident diagrams (to make certain they occurred on the approach in question), the approach was removed from the database to ensure the accuracy of approach-assigned accidents counts. This reduced our sample of 460 approaches (115 intersections containing 4 approaches) to 365 approaches. Over the four-year study period on these approaches, there were 589 rear-end accidents.

Traffic flow data were gathered from annual site surveys and highway census data. Traffic control data were extracted from corresponding database documents and roadway and environmental data were also gathered. In addition a measure of visual noise was developed and included in the database. This is potentially relevant because previous studies (e.g., Miura 1992) have shown that increasing complexity in the driving environment results in increased reaction times. To account for the effect of driving environment complexity, an index of visual noise level 
(with values ranging from 0 to 4 ) is used in this study. To evaluate the visual noise level, a site survey of intersections was conducted by trained survey personnel. They were asked to judge visual noise level of the surveyed intersection approaches on a scale from 0 to 4 with: level 0 being areas of isolated residential houses and factories; level 1 being residential areas of concentrated multistory residential houses; level 2 being mixed residential areas and general office districts; level 3 being concentrated office areas; and level 4 being prosperous commercial areas typically near railway stations.

Table 1 provides summary statistics for select continuous variables in the database and Table 2 provides frequency results for select integer variables in the database. To help with interpretation of these variables, Figure 3 is provided. In this figure, the entering approach is the one being studied for accident frequency and the others set up left, right, and opposite designations. If data are complete, each intersection will generate four observations, with each approach becoming the entering approach for one observation.

\section{ESTIMATION RESULTS}

Model estimation results are shown in Table 3. A total of 19 explanatory variables were found to significantly affect the rear-end accident probability. Of these 19 variables, 13 had 
impacts on the probability of encountering an obstacle vehicle and 6 had impacts on driver failure probabilities. Table 3 shows that overall model fit was good as represented by $\rho^{2}$ which is calculated as one minus the ratio of the log-likelihood function at convergence (all coefficients estimated) over the log-likelihood function with only constants estimated. The sign of each coefficient shows the effect, either increasing (positive) or decreasing (negative) the probability of a rear-end accident.

Turning first to factors affecting the probability of encountering an obstacle vehicle, $P_{o}$, eight explanatory variables were found to decrease the rear-end accident probability and five were found to increase it. Approaches with higher speed limits, signal progression, and elevated roads above them lowered the probability of encountering an obstacle vehicle, $P_{o}$. Some caution should be exercised in interpreting the speed limit finding because roadways may have higher speed limits because they are safer. Thus the negative effect that higher speed limits have on the probability of encountering an obstacle vehicle may reflect this. Signal progression clearly has a smoothing effect on traffic and this is reflected in the reduced probability that an obstacle vehicle will be encountered. The elevated road finding may be an artifact of the data with such intersections having fewer environmental disturbances.

The reciprocal of the number of approach lanes was also found to significantly decrease the probability of encountering an obstacle vehicle. This result implies that an increase in the number 
of lanes will increase the probability of encountering an obstacle vehicle (the variable will become smaller and, hence, will increase $P_{o}$ ). This may be due to the increasing conflict frequency between through traffic and opposite right-turn traffic when the number of lane in the entering approach increases.

The finding that intersections located in central business district (CBD) lowered the probability of encountering an obstacle vehicle is consistent with the findings of Poch and Mannering (1996). They obtained the same result when analyzing intersection rear-end accident frequency on a monthly basis using standard negative binomial regression. This finding can be contributed to a number of factors including traffic calming efforts to improve traffic safety and stricter enforcement of traffic regulations in $\mathrm{CBD}$ areas.

The total number of lanes in the left approach (see Figure 3 for a graphic) was found to decrease the probability of an obstacle vehicle. With more lanes in the left approach, conflicts between left-turn flow and opposite right-turn flow are reduced. Thus, through-vehicle decelerations caused by opposite right-turn flow will go down correspondingly and this is reflected in the coefficient estimate.

Approaches with a median fence and four-phase signal control were found to lower the probability of encountering an obstacle vehicle. The existence of median fences can prevent pedestrians from crossing illegally thus reducing disturbances that may cause an obstacle vehicle. 
The four-phase control reduces conflicts between through-traffic flow and opposite right-turn traffic flow and this is reflected in the estimation results.

When the angle between the entering approach and the left approach was less than 105 degrees (see Figure 3 for an illustration of entering and left approaches), the likelihood of encountering an obstacle vehicle increased. This is likely a reflection of the more complex turning movements associated with smaller-angle approach configurations. In addition, the volume of turning vehicles, including right-turning and left-turning vehicles on the entering approach and right-turning vehicles on the opposite approach, increased the probability of encountering an obstacle vehicle. Finally, the higher the average time headway of the entering approach's traffic, the greater the probability of encountering an obstacle vehicle. This result was unexpected. One possible explanation may be that longer headway time intervals in the data encourage more opposite right-turn vehicle movements as well as signal-disregarding pedestrian movements. Applications of the model to other data may cast additional light on this finding.

Turning to factors found to affect drivers' failure probabilities, $P_{f}$, the total number of lanes in the entering approach was found to reduce failure probabilities. This likely relates to the wider vision field provided by a higher number of lanes. In contrast, the greater the number of intersection approaches sheltered by elevated roadways, the greater the probability of drivers' failure probabilities. It is speculated that different light conditions may affect perception times in 
this regard.

As expected, the 0 to 4 scale of visual noise was found to significantly increase driver failure probabilities indicating that driver distractions significantly increase perception times. The night-to-day flow ratio (defined as nighttime traffic volume divided by daytime traffic volume) was found to increase driver failure probabilities. This finding reflects increased perception times (and possibly reaction times) during nighttime conditions. Because critical traffic volumes are directly controlled for elsewhere in the model with variables such as right- and left-turn volumes and headways, the night-to-day flow ratio is simply providing an overall increase in accident likelihood when traveling at night.

Higher speed limits and higher slopes of the entering approach were also found to increase driver failure probabilities. Because vehicle speed is proportional to stopping distance, it is clear that higher speeds increase driver failure probabilities. Slopes of the entering approach can affect both stopping distance (downhill) and ease of perception (uphill). We were unable to find significantly different effects between uphill and downhill slopes and their effects are thus combined into a single variable. Finally, the coefficient estimate for reciprocal of negative binomial dispersion parameter $(\theta)$ is significant indicating that they data are over dispersed and the negative binomial assumption was correct (as opposed to a Poisson).

To get some sense of the relative probabilities of encountering an obstacle vehicle and 
drivers' failure probabilities, probabilities were calculated at all approaches. The average probability of encountering an obstacle vehicle, $P_{o}$ was 0.339 and the average probability of a following vehicle driver's failure, $P_{f}$ was only 0.0000002 . As expected, the likelihood of encountering an obstacle vehicle is much higher than the probability of driver failure which is reasonable because traffic flow is frequently interrupted by disturbances, but rear-end accidents are still relatively rare because drivers' available perception/reaction time is almost always greater than the needed perception/reaction time.

\section{SUMMARY AND CONCLUSIONS}

Because intersections are accident-prone areas, there is a clear need to analyze intersection accident data to determine factors causing accidents and the potential effectiveness of countermeasures. This paper presents a new model based on the occurrence mechanism of rear-end accidents at intersections. Using data from hundreds of intersection approaches, the occurrence of rear-end accidents was studied considering the probability of encountering an obstacle vehicle and the probability of a driver failing to react quickly enough to avoid a collision with the obstacle vehicle.

In our model, the probability of encountering an obstacle vehicle is assumed to be a 
function of the frequency of disturbances that cause the driver of a leading vehicle in a vehicle pair to decelerate. The probability of the trailing vehicle's driver failing to respond is the probability that this drivers' needed perception/reaction time is less than the available perception/reaction time.

By considering the occurrence mechanism of rear-end accidents, the model can explicitly account for human factors. Moreover, by considering factors that affect both the probability of encountering an obstacle vehicle and the probability of a driver failing to react quickly enough to avoid a collision with the obstacle vehicle, explanatory variables can be significant in both functions and sometimes affect overall accident probability different directions. An example is the effect of the speed limit that was found to decrease the probability of encountering an obstacle vehicle, but increase the probability of a driver failure. Existing models with just canonical linear or log-linear link functions are unable to account for such dual impacts of important explanatory variables.

In terms of future work, there is a need to asses the temporal and spatial transferability of our proposed modeling approach. With additional data from other geographic locations, this model has the potential to provide important new insights into rear-end accident occurrence. 


\section{ACKNOWLEDGEMENTS}

This research was funded under a grant from the Kyoei Mutual Fire and Marine Insurance Company. The authors would like to thank Mr. Akiyama Hisao, Mr. Hanada Kenji, Mr. Shiina Yasuo and Mr. Shiina Hiroo of Tokyo Metropolitan Police Department for their active corporation during this study.

\section{REFERENCES}

Bates, J.T. (1995). Perception reaction time, ITE Journal, Vol. 65, No. 2, pp35-36.

Carson, J. and Mannering, F. (2001). The effect of ice warning signs on accident frequencies and severities, Accident Analysis and Prevention, 33(1), 99-109.

Hauer, E., Ng, J., and Lovell, J. (1988). Estimation of Safety at Signalized Intersections, Transportation Research Record 1185, 48-60.

Institute for Traffic Accident Research and Data Analysis (1996). Traffic Statistics, Tokyo (in Japanese).

Johansson, G. and Rumar, K. (1971). Drivers' Brake Reaction Times, Human Factors, 13(1), 23-27. 
Jovanis, P. P., and Chang, H. (1986). Modeling the Relationship of Accidents to Miles Traveled, Transportation Research Record 1068, 42-51

Kao, J. H. K. (1960). A summary of some new techniques in failure analysis, Proceedings of Sixth National Symposium on Reliability and Quality Control, Washington DC, 190-201.

Lawless, J. F. (1987). Negative binomial and mixed Poisson regression, The Canadian Journal of Statistics, 15(3), 209-225.

Mannering, F. and W. Kilareski (1998) Principles of Highway Engineering and Traffic Analysis, John Wiley \& Sons, New York, NY (2nd Edition), 340 pages.

Miaou, S.-P. and Lum H. (1993). Modeling vehicle accidents and highway geometric design relationships, Accident Analysis and Prevention, 25(6), 689-709.

Miaou, S., Hu, P.S., Wright, T., Rathi, A.K., and Davis, S.C. (1992). Relationship between truck accidents and highway geometric design: a Poisson regression approach, Transportation Research Record 1376, 10-18.

Milton, J. and Mannering, F. (1998). The relationship among highway geometrics, traffic-related elements and motor-vehicle accident frequencies, Transportation 25(4), 395-413. 
Miura T. (1992). Visual Search in Intersections, IATSS Research, 16(1), 42-49.

Olson, P.L., and Sivak, M. (1986). Perception-response time to unexpected roadway hazards, Human Factors, 28(1), 91-96.

Pitman J. (1993). Probability, Springer-Verlag New York, Inc.

Plait, A. (1962). The Weibull distribution - with the tables, Industrial Quality Control, 19(1), 17-26.

Poch, M., and Mannering F. (1996). Negative binomial analysis of intersection accident frequencies, Journal of Transportation Engineering, 122(2), 105-113.

Shankar, V., Mannering, F., and Barfield, W. (1995). Effect of roadway geometrics and environmental factors on rural freeway accident frequencies, Accident Analysis and Prevention, 24(4), 425-436.

Shankar, V., J. Milton, and F. Mannering (1997). Modeling accident frequencies as zero-altered probability processes: an empirical inquiry, Accident Analysis and Prevention, 29(6), 829-837.

Welford, A.T. (1977). Motor performance, in J.E. Birren and K.W. Schaie (Eds.), Handbook of 
the psychology of aging, Van Nostrand, New York.

\section{NOTATION}

The following symbols are used in this paper:

$$
\begin{aligned}
\mathrm{E}(\cdot) & =\text { expected value } \\
e & =\text { exponential function } \\
\varepsilon & =\text { error term } \\
f & =\text { function } \\
m & =\text { Poisson distribution parameter } \\
n & =\text { number of accidents } \\
t & =\text { time } \\
L(\cdot) & =\text { log-likelihood function } \\
P(\cdot) & =\text { probability of having a specific number of accidents } \\
P & =\text { probability } \\
v & =\text { traffic volume } \\
\mathrm{X} & =\text { vector of explanatory variables for } P_{o} \\
Z & =\text { vector of explanatory variables for } P_{f} \\
x & =\text { specific explanatory variable } \\
\delta & =\text { variance of gamma-distributed error term (negative binomial } \\
\beta & \text { dispersion parameter) } \\
\beta & =\text { vector of estimable coefficients for } P_{o} \\
\phi & =\text { vector of estimable coefficients for } P_{f}
\end{aligned}
$$




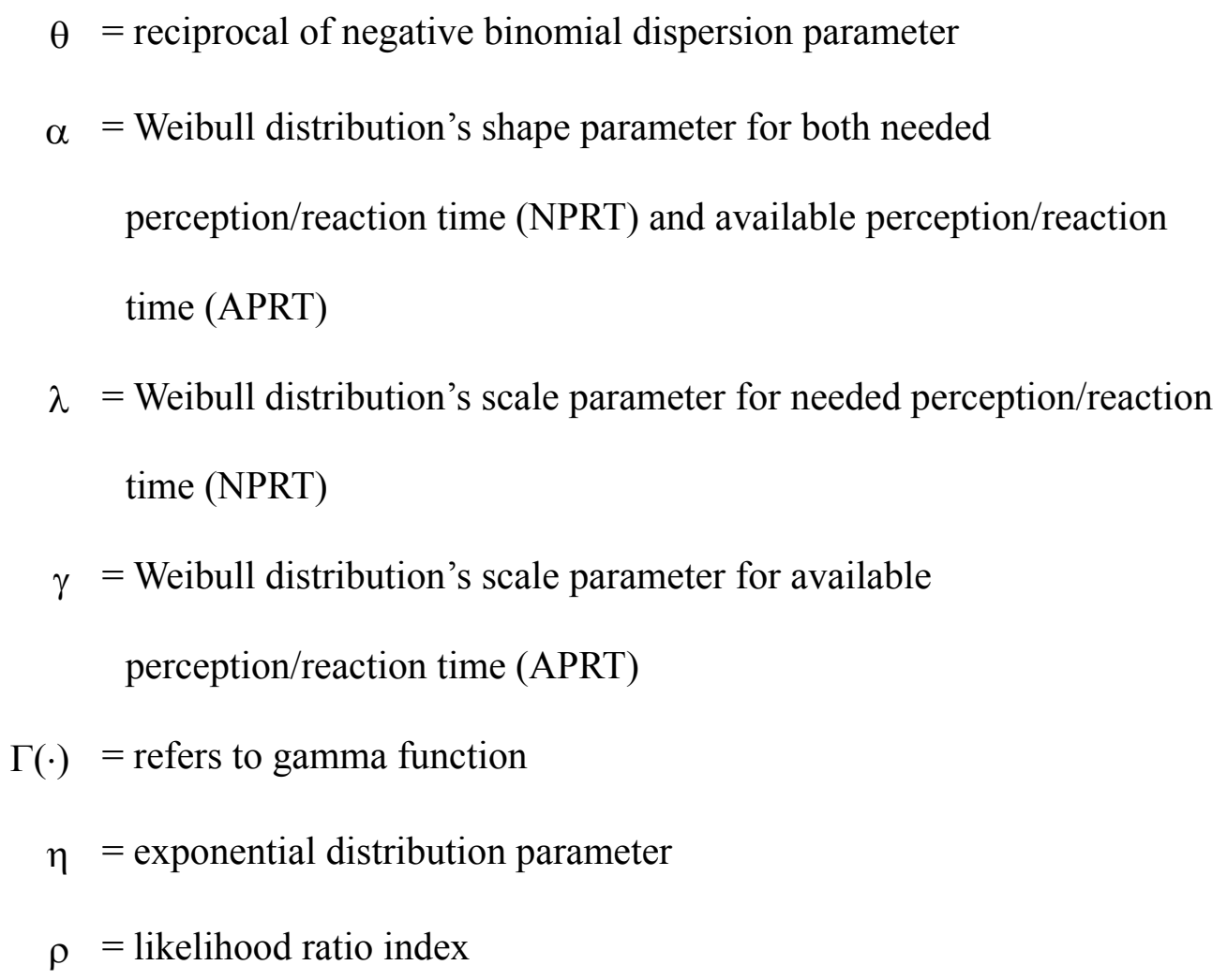

The following subscripts are used in this paper:

$$
\begin{aligned}
& a=\text { abbreviation for "available" } \\
& i=\text { intersection code } \\
& j=\text { disturbance type } \\
& k=\text { leg code ranges from } 1 \text { to } 4 \\
& o=\text { obstacle vehicle } \\
& f=\text { response failure } \\
& h=\text { human factor }
\end{aligned}
$$




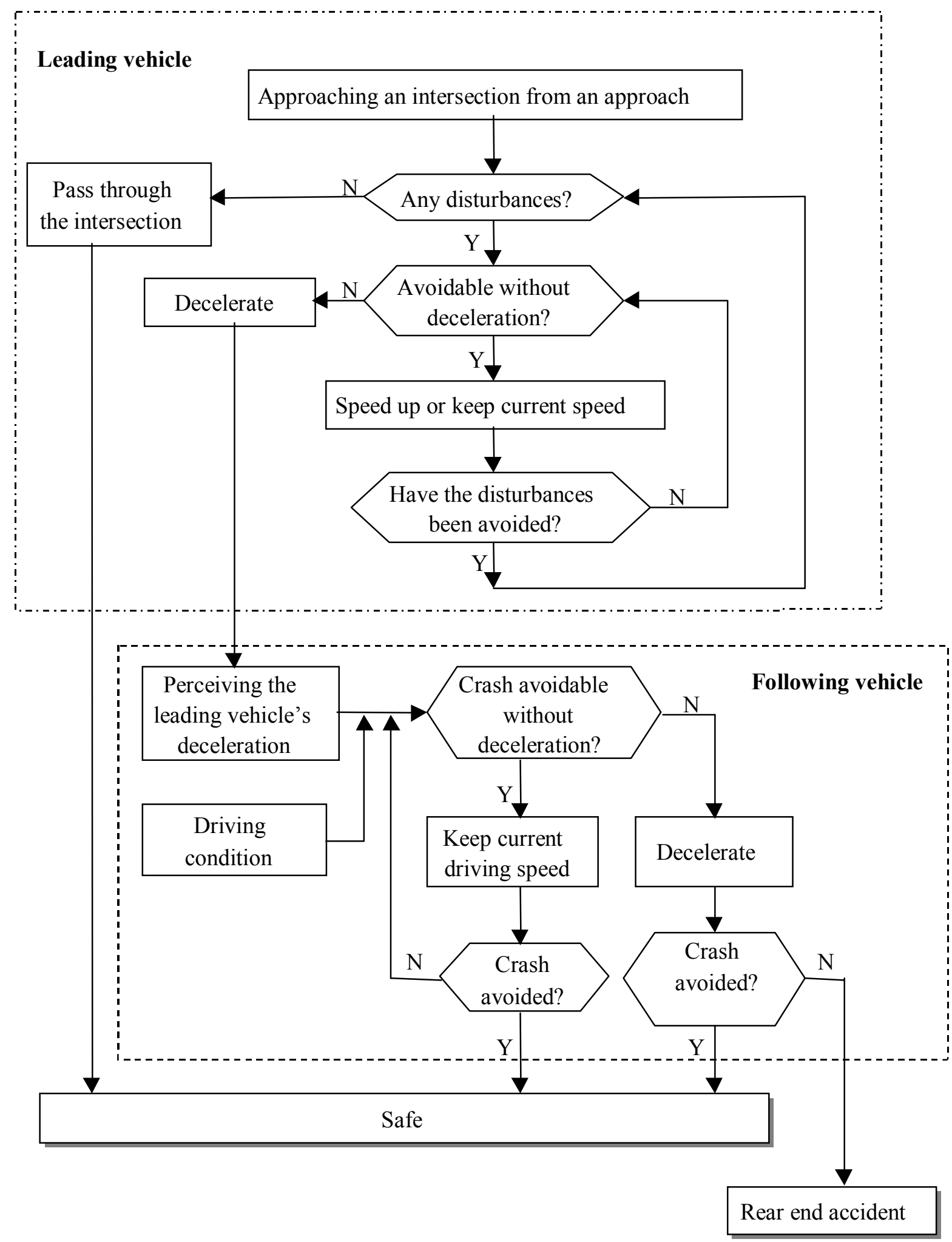

Fig. 1. Flow chart of rear-end accident causation. 


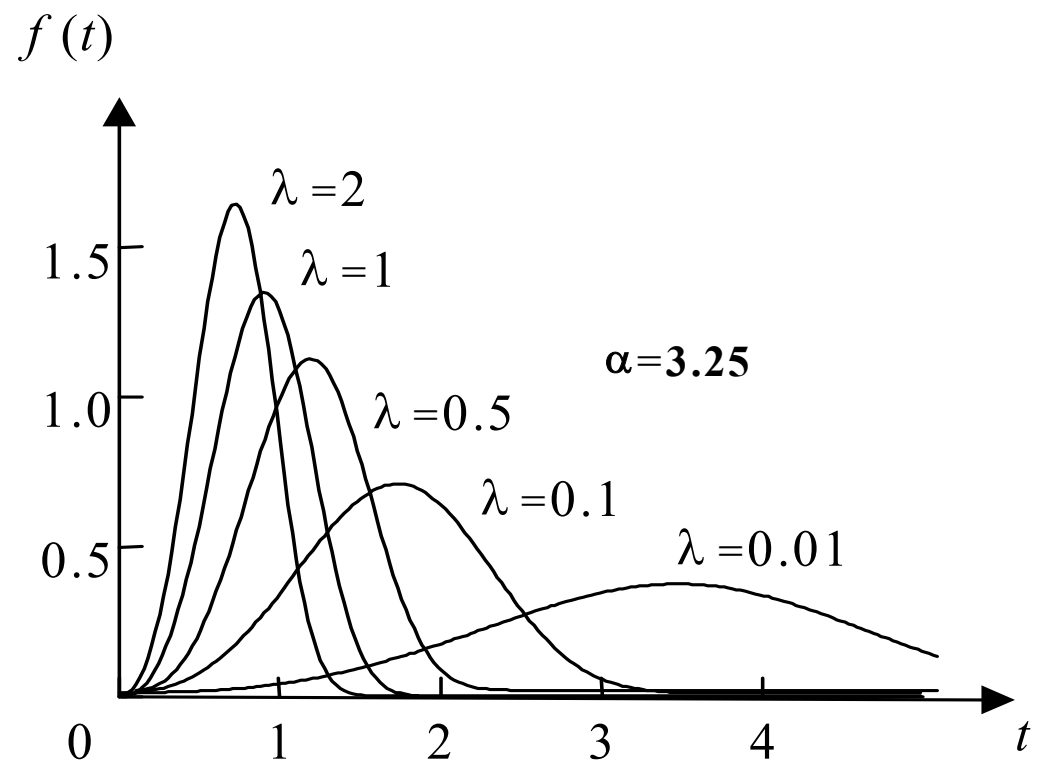

Fig. 2. Weibull density functions with different scale parameters, $\lambda$, and constant shape parameter, $\alpha$. 


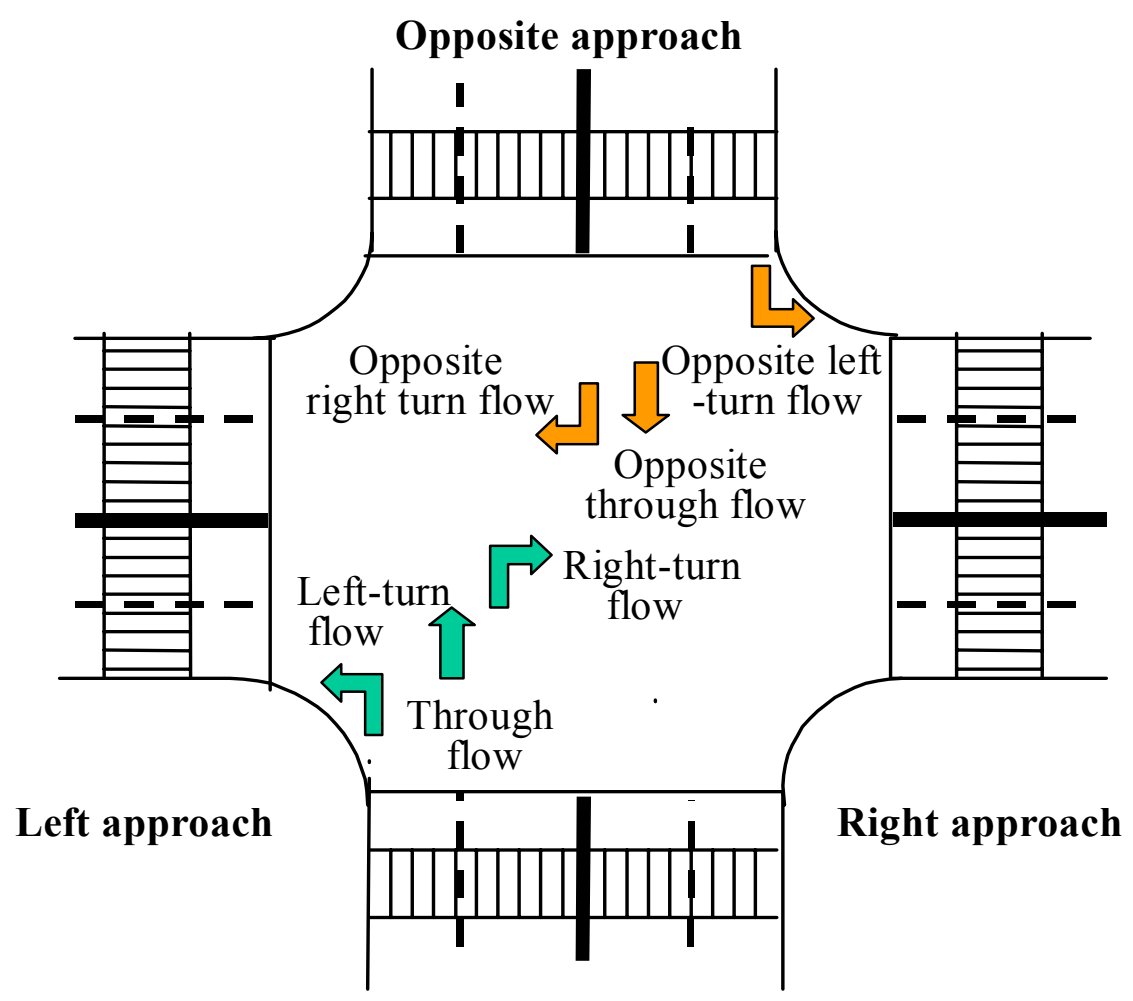

Entering approach

Fig. 3. Graphic of intersection approaches. 
Table 1. Summary statistics for continuous variables.

\begin{tabular}{|c|c|c|c|c|}
\hline Variable & Mean & $\begin{array}{l}\text { Standard } \\
\text { Deviation }\end{array}$ & Minimum & Maximum \\
\hline Rear end accidents per approach & 1.61 & 1.99 & 0.00 & 11.00 \\
\hline Daily left-turn traffic volume of the entering approach & 3,109 & 2,347 & 0 & 20,467 \\
\hline Daily through traffic volume of the entering approach & 12,844 & 9,088 & 142 & 51,660 \\
\hline Daily right-turn traffic volume of the entering approach & 3,139 & 2,244 & 0 & 11,928 \\
\hline Speed limit of the entering approach $(\mathrm{km} / \mathrm{h})$ & 49.37 & 8.83 & 30 & 60 \\
\hline Total number of approach lanes (including both entering & 4.96 & 1.88 & 1.00 & 11.00 \\
\hline lanes and exiting lanes) & & & & \\
\hline $\begin{array}{l}\text { Total number of lanes in left approach (including both } \\
\text { entering lanes and exiting lanes) }\end{array}$ & 5.02 & 1.94 & 1.00 & 12.00 \\
\hline Night-to-day traffic flow ratio & 0.49 & 0.06 & 0.33 & 0.78 \\
\hline
\end{tabular}


Table 2. Frequency results for integer variables.

\begin{tabular}{|c|c|c|c|c|c|}
\hline \multirow{2}{*}{ Variable } & \multicolumn{5}{|c|}{$\begin{array}{l}\text { Number of observations } \\
\text { for each value }\end{array}$} \\
\hline & 0 & 1 & 2 & 3 & 4 \\
\hline $\begin{array}{l}\text { Signal progression ( } 1 \text { if with signal progression, } 0 \\
\text { otherwise) }\end{array}$ & 183 & 182 & - & - & - \\
\hline $\begin{array}{l}\text { Number of intersection approaches sheltered by elevated } \\
\text { roadways }\end{array}$ & 277 & 82 & 6 & - & - \\
\hline $\begin{array}{l}\text { Intersection location ( } 1 \text { if in central business district } \\
\text { (CBD), } 0 \text { otherwise) }\end{array}$ & 165 & 200 & - & - & - \\
\hline $\begin{array}{l}\text { The existence of fence median of the entering approach } \\
\text { (1 if there is, } 0 \text { otherwise) }\end{array}$ & 188 & 177 & - & - & - \\
\hline Signal control pattern ( 1 for 4 phase control, 0 otherwise) & 111 & 254 & - & - & - \\
\hline $\begin{array}{l}\text { Angle of the entering approach and left approach ( } 1 \text { if } \\
\text { less than } 105^{\circ}, 0 \text { otherwise) }\end{array}$ & 276 & 89 & - & - & - \\
\hline Visual noise (ranging from 0 to 4 ) & 64 & 101 & 131 & 46 & 23 \\
\hline $\begin{array}{l}\text { Slope of the entering approach ( } 1 \text { if greater than } \pm 3 \% \text {, } \\
0 \text { otherwise) }\end{array}$ & 339 & 26 & - & - & - \\
\hline
\end{tabular}


Table 3. Model estimation results.

\begin{tabular}{lcc}
\hline & Estimated & Standard \\
Variable & Coefficient & error p-value \\
& & \\
\hline
\end{tabular}

\section{Variables affecting the probability of encountering an}

\section{obstacle vehicle}

Constant

Speed limit of the entering approach in $\mathrm{km} / \mathrm{h}$

Signal progression ( 1 if with signal procession, 0 otherwise)

Number of intersection approaches sheltered by elevated

roadways

Reciprocal of number of lanes of the approach

Intersection location ( 1 if in central business district (CBD),

0 otherwise)

Total number of lanes in left approach (including both entering lanes and exiting lanes)

The existence of fence in median of the entering approach ( 1 if there is, 0 otherwise)

Signal control pattern ( 1 for 4 phase control, 0 otherwise)

Angle of the entering approach and left approach (1 if less than $105^{\circ}, 0$ otherwise)

Right-turn volume in thousands of vehicles (4 years) of the

Right-turn volume in thousands of vehicles (4 years) of the opposite approach

4.236

1.810

0.020

$-0.064$

0.028

0.025

$-0.254$

0.223

0.257

$-3.653$

0.558

0.000

$-7.821$

0.259

0.001

$-0.487$

0.059

0.062

$-0.093$

0.057

0.118

$-0.218$

0.208

0.295

$-0.431$

0.268

0.108

0.566

0.205

0.007

0.073

0.036

0.044

0.249

0.048

0.000 
entering approach

Left-turn volume in thousands of vehicles (4 years) of the

0.137

0.045

0.002

entering approach

Average time headway in seconds of entering approach's

0.053

0.013

0.000

through traffic

\section{Variables affecting drivers' failure probability}

Constant

Total number of approach lanes (including both entering lanes and exiting lanes)

Number of intersection approaches sheltered by elevated roadways

Visual noise (ranging from 0 to 4 )

Night-to-day traffic flow ratio

Speed limit of the entering approach (in $\mathrm{km} / \mathrm{h}$ )

Slope of the entering approach (1 if greater than $\pm 3 \%$,

0 otherwise)
$-19.455$

$-0.441$

2.546

0.437

0.000

0.130

0.086

1.588

0.043

0.622

0.173

0.934

0.016

0.090

0.000

\section{Other estimate}

Reciprocal of negative binomial dispersion parameter $(\theta=1 / \delta)$

3.708

1.094

0.001

Number of observations 
Likelihood ratio index, $\rho^{2}$

0.698 


\section{List of Figures}

Fig. 1. Flow chart of rear-end accident causation.

Fig. 2. Weibull density functions with different scale parameters, $\lambda$, and constant shape parameter, $\alpha$.

Fig. 3. Graphic of intersection approaches. 